\title{
Steimann Pin Repair of Zygomatic Complex Fractures*
}

\author{
Jonathan B. Salinas, ${ }^{1,2 \#}$, Darshni Vira ${ }^{1,2}$, David Hu ${ }^{1}$, David Elashoff ${ }^{3,4}$, \\ Elliot Abemayor ${ }^{1,2}$, Maie St. John ${ }^{1,2,3}$ \\ ${ }^{1}$ Department of Head and Neck Surgery, David Geffen School of Medicine at University of California, Los Angeles, USA \\ ${ }^{2}$ Harbor-UCLA Medical Center, Los Angeles, USA \\ ${ }^{3}$ Jonsson Comprehensive Cancer Center, Los Angeles, USA \\ ${ }^{4}$ Department of Medicine, David Geffen School of Medicine at University of California, Los Angeles, USA

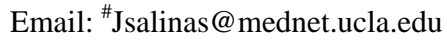

Received February 8, 2013; revised March 12, 2013; accepted March 19, 2013

\begin{abstract}
Purpose: To present the treatment of zygomaticomaxillary complex (ZMC) fractures with closed-reduction Steinmannpin fixation and to compare it to the reduction and aesthetic outcomes of open-reduction techniques (ORIF). Materials and Methods: Case series. Charts for 23 patients with ZMC fractures presenting to the Head and Neck Surgery Department at Harbor-UCLA Medical Center from 2005 to 2009 were reviewed. Pre- and post-operative computed tomography (CT) scans were analyzed. Follow up ranged from 3 to 55 months. Interviews were conducted to evaluate the patient's satisfaction. Patients were placed in two groups: those treated with ORIF and those treated with closed-reduction Steinmann-pin fixation. Results: Twelve patients had complete data for analysis. Average operative time was significantly lower for patients treated with closed-reduction as compared to open-reduction: 65.3 minutes vs. 162.5 minutes $(\mathrm{p}=0.02)$. Bony realignment and aesthetic results were comparable in both groups. Additionally, only one $1 \mathrm{~cm}$ facial incision was required with this repair system versus several incisions using traditional methods. Conclusions: Closed-reduction Steinmann-pin fixation of ZMC fractures provides adequate bony alignment and aesthetics. Our study supports this system in the repair of ZMC fractures as it requires significantly less operating time, one small incision, and excellent patient outcomes.
\end{abstract}

Keywords: Steimann Pin; Zygomatic Complex Fracture; Zygomaticomaxillary Complex Fracture

\section{Introduction}

The etiology of facial fractures differs from one country to another worldwide. In addition, statistics on the main sources responsible for the injury differs depending on the prevailing socioeconomic, cultural, and environmental factors [1-3]. Earlier studies listed traffic accidents as the major etiological factor of maxillofacial injuries. [1] Other incidents such as assaults, falls, sports-related injuries, industrial, work accidents, civilian warfare, and animal attacks have been noticed to be less likely to cause head injuries [2,4]. Recent research has shown that assault has replaced motor vehicle accidents as the most common cause of maxillofacial fractures [5]. Nevertheless, motor vehicle accidents continue to present a fre quent mechanism for facial fractures.

Alterations to the form and function of the face due to injury can lead to a significant change in the perceptions of how a patient feels, interacts and reacts to a social

\footnotetext{
*There are no potential conflicts of interest, including any financial support of this work.

${ }^{\#}$ Corresponding author.
}

environment. Therefore, it is important for the surgeon to repair both the soft-tissue injury and the bony infrastructure to the patient identity. Technology has allowed for significant improvement in the treatment of these injuries. Resources such as computed tomography, three-dimensional reconstructions, plate-and-screw fixation, and bone grafting give the surgeon a greater ability to restore the bony structures [6].

Similarly, it is important to recognize that zygomaticomaxillary fractures have been associated with hyperesthesia (52.2\%), trismus (47.3\%), diplopia (8.3\%) and malocclusion (5.3\%) in the preoperative period [7]. Therefore, prompt recognition and treatment of the injury is important to decrease any potential long term sequelae.

The contour of the midfacial area is formed by the zygomatic bone [8]. This bone is a protruded, three-dimensional structure that is susceptible to injury from trauma, with resulting facial asymmetry. Since the zygomatic arch serves as the origin of the masseter on its inferior margin [9], trismus can present a functional problem in case of injury to the zygomatic bone [1]. Another functional consideration is the fact that the anterior portion of 
the lateral orbital wall is formed by the zygoma. As many as $65 \%$ of patients with injury to the zygomatic arch may present with enophthalmos, while $49 \%$ may present with diplopia [1,9]. Therefore, accurate reduction and rigid fixation of fractured sites are extremely important for aesthetic and functional outcomes.

In order to expose the fractured zygomatic bone, approaches such as supraorbital brow incision, subciliary incision, lateral brow incision and intraoral incision can be performed [8,10-14]. Following this, an appropriate reduction of the bone segment fractured and displaced should be performed, allowing for the correction of facial asymmetry through rigid fixation under direct vision.

Open reduction of these fractures allows for the gross confirmation of the reduction of the fracture site and rigid fixation [14]. However, open reduction can lead to undesirable sequelae such as ectropion, facial nerve injury and drooping of the cheek owing to extensive dissection of the periosteum $[8,15]$. Moreover, as maxillofacial fractures frequently occur in young persons, the facial incisions can be a burden to surgeons and patients due to unsightly postoperative scars. In the same way, metal plates and screws used for fixation may require removal at a later date, translating into further surgery, which could potentially increase scarring [16,17].

Based on recent reports, the most significant types of chronic residual sequelae of zygomatic bone fracture are deformities resulting from mal reduction of the zygomatic prominence, enophthalmos, cheek anesthesia or dysesthesia, and trismus [18]. Of note, enophthalmos can be a consequence of the enlargement of the orbital cavity, soft tissue, or lower lid retraction [18].

In order to avoid the complications related to an open reduction approach, a closed reduction approach can be performed. The use three-dimensional computed tomography (CT) before surgery, can help in determining the severity of zygoma complex fractures and the displacement of bone segments in the preoperative period without the need for an open procedure $[8,15]$. In the same way, a preoperative three-dimensional CT can be used to detect the direction to which the extrinsic impact was exerted and the bone fragments were displaced, during the injury. Based on this information one can attempt to reduce the fracture in the direction the extrinsic impact was exerted, allowing for an appropriate and simple correction, as compared with other methods $[15,19]$. The use of a Steinmann pin can provide appropriate fixation of the bone segments. Rinehart et al. describe experiments using cadaveric skulls, reporting that 2-point fixation of the zygomaticofrontal and zygomaticomaxillary areas is effective [20]. Furthermore, Abemayor et al. showed that repair of selected unstable malar fractures with immobilization using a Kirschner wire can be used with low morbidity as well as outstanding cosmetic and functional results [21]. Finally, the Steinmann pin's lever movement allows for a closed reduction while considering the width and height of the midfacial complex [15].

This case series presents our experience treating ZMC fractures through closed reduction using a Steinmann pin.

\section{Methods and Materials}

This study was carried out under Institutional Review Board (IRB) approval. Charts for 23 consecutive patients with ZMC fractures presenting to the OtolaryngologyHNS Department at Harbor-UCLA Medical Center from 2005 to 2009 were reviewed. Postoperative CT scans were reviewed by a single head and neck radiologist and analyzed for malar symmetry.

Patient Selection: Only patients with type B fractures were included in this study. Patients with other midface fractures were also excluded. Patients who revealed ocular symptoms and/or major depression of the orbital floor necessitating manipulation of the orbital floor were also excluded because a lower lid approach and open reduction with or without free bone transplantation is usually required in such cases, and the influences of intervention should be discussed separately. Based on this criteria eleven patients were excluded. Therefore, twelve patients with type B fractures treated with internal or external fixation had appropriate data for analysis. Patients were separated into two groups: those treated with open reduction and internal fixation (ORIF), and those treated with closed reduction and transzygomatic external fixation (Steinmann Pin). All patients were taken to surgery 3 14 days post-trauma.

Closed reduction and transzygomatic external fixation using Steinman pin: After patients were nasally intubated, general anesthesia was induced. The entire face was then prepped and draped in the standard sterile fashion. The temporal region was infiltrated with $1 \%$ lidocaine with 1:100,000 epinephrine. A \#15 blade was then used to make a 1-cm Gilles incision posterior to the temporal hairline. This incision was taken down to the temporalis fascia and temporalis muscle. A key elevator was then placed in the incision and used to elevate the zygomatic arch into place. Adequate reduction was confirmed with palpation of the fractured site. Once the fracture was reduced appropriately, a small facial incision at the level of the cheek was made in order to place a 3.2-mm Steinmann pin. The pin was then drilled in the direction of the hard palate until palpation confirmed its position through the hard palate. Next, the pin was cut flush at the level of the skin, and the skin incision was closed using a 6-0 nylon suture in an interrupted fashion. At the same time, bone wax was applied to cover the intraoral end of the pin to improve patient comfort. The Gilles incision was 
closed also using a 6 - 0 nylon suture in an interrupted fashion.

Open Reduction Internal Fixation: After patients were nasally intubated, general anesthesia was induced. The entire face was then prepped and draped in the standard sterile fashion. The gingivobuccal sulcus was infiltrated with 1\% lidocaine with 1:100,000 epinephrine. A \#15 scapel was used to make a gingivobuccal sulcus incision. Next, the face of the maxilla was degloved up to the level of the infraorbital rim. A blunt elevator was then inserted laterally beneath the zygomatic arch and an attempt at reduction was made. In cases where it was difficult to assess the degree of reduction, further exposure was necessary. The exposure was usually made by a lower eyelid incision via a transconjunctival approach. The intraoral incision was also used to facilitate the lower eyelid dissection, allowing for the fractured fragment to be reduced in a more anatomical position. In the same way, adequate reduction was confirmed with palpation of the fractured site. Once the fracture was aligned, the infraorbital rim was typically plated with a $1.5-\mathrm{mm}$ plate. Next, a 2-mm L-plate is placed along the zygomaticomaxillary buttress laterally. All incisions were closed in the standard fashion.

Postoperative follow-up ranged from three to 61 months. Telephone interviews were conducted to evaluate patient satisfaction with aesthetic outcome and surgical complications, including hyperesthesia, diplopia, trismus, and malocclusion. All patients who returned for follow up were asked to obtain a post-operative can 3 to 8 weeks after the procedure, depending on when they were able to come for their follow up appointment.

Operative time was compared between the groups using a Wilcoxon rank sum test. Statistical analysis was performed using S-plus (version 8, TIBCO Software Inc, Palo Alto, CA). P-values less than 0.05 were considered significant.

\section{Results}

Of the twenty-three patients, twelve patients had sufficient data for analysis. A total of six patients were found to have undergone ZMC fracture repair by open reduction with internal fixation (ORIF) (Table 1). In the same way, six patients had undergone ZMC fracture repair via closed reduction with a Steinmann Pin (Table 1). The ages of the patients who received ORIF repair and closed reduction repair were similar with means of 40.3 and 40.2 years, respectively. The ages ranged from 22 to 58 years for ORIF repair and from 18 to 56 years for closed reduction repair ranged (Table 1). The operative time of the ORIF repairs ranged from 62 to 313 minutes, and the operative time for the Steinmann pin repairs ranged from 40 to 120 minutes (Table 1). Average operative time was significantly ( $p=0.02$ ) lower for patients treated via the closed technique as compared to the open technique: 65.3 minutes and 162.5 minutes (Table 2). Additionally, only a single one-centimeter incision was required with the closed-repair system versus several incisions using traditional methods. After reviewing the CT scans with a Head and Neck Radiologist, it was determined that the bony alignments were appropriate in patients from both groups (Figures 1 and 2). Based on telephone interviews, patients were found to be satisfied with the aesthetic and functional results after the ZMC fracture repair.

\section{Discussion}

The zygoma comprises the lateral aspect of the mid-facial skeleton, shaping the lateral and inferior rim, as well as the malar eminence [1]. There are articulations of these facial projections with the sphenoid bone in the lateral orbit, the frontal bone superiorly, the maxilla medially, and the maxillary alveolus inferiorly [1]. Its prominent projection makes the zygoma susceptible to traumatic injury.

Table 1. Demographics and operative time for ZMC repairs using ORIF and steinmann pin.

\begin{tabular}{|c|c|c|c|c|}
\hline Patient & Age (y) & Gender & Surgical Technique & $\begin{array}{l}\text { Operative } \\
\text { Time (m) }\end{array}$ \\
\hline 1 & 49 & M & ZMC ORIF $^{*}$ & 313 \\
\hline 2 & 51 & M & ZMC ORIF & 110 \\
\hline 3 & 58 & $\mathrm{~F}$ & ZMC ORIF & 150 \\
\hline 4 & 37 & M & ZMC ORIF & 62 \\
\hline 5 & 22 & M & ZMC ORIF & 170 \\
\hline 6 & 25 & M & ZMC ORIF & 170 \\
\hline 7 & 54 & M & ZMC Steinmann Pin & 55 \\
\hline 8 & 18 & M & ZMC Steinmann Pin & 77 \\
\hline 9 & 26 & M & ZMC Steinmann Pin & 120 \\
\hline 10 & 46 & M & ZMC Steinmann Pin & 55 \\
\hline 11 & 41 & M & ZMC Steinmann Pin & 40 \\
\hline 12 & 56 & M & ZMC Steinmann Pin & 45 \\
\hline
\end{tabular}

$\mathrm{y}=$ years; $\mathrm{m}=$ minutes; ${ }^{*}=$ concurrent mandibular fracture repair.

Table 2. Operative time average for $\mathrm{ZMC}$ repairs using ORIF and steinmann pin.

\begin{tabular}{cc}
\hline Surgical Technique & Operative Time Average (m) \\
\hline ZMC ORIF & $162.5^{*}$ \\
ZMC Steinmann Pin & $65.3^{*}$ \\
\hline
\end{tabular}

$\mathrm{m}=$ minutes; ${ }^{*}$ Statistical analysis using S-plus yields a significant result ( $\mathrm{p}=$ 0.02). 


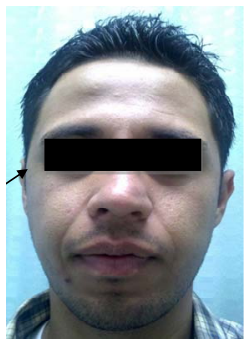

(a)

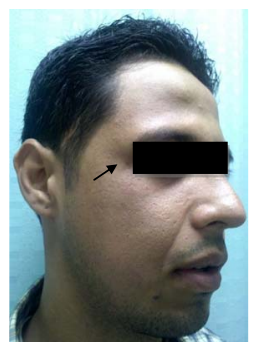

(b)

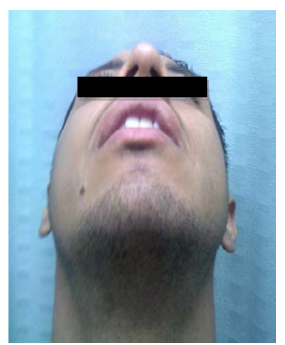

(c)
Figure 1. Postoperative photographs 9 months after ZMC fracture repair using a Steinmann pin. Note a well-healed scar lateral to the right eye (Black arrow). (a) Frontal view; (b) Right oblique view; (c) Basal view.
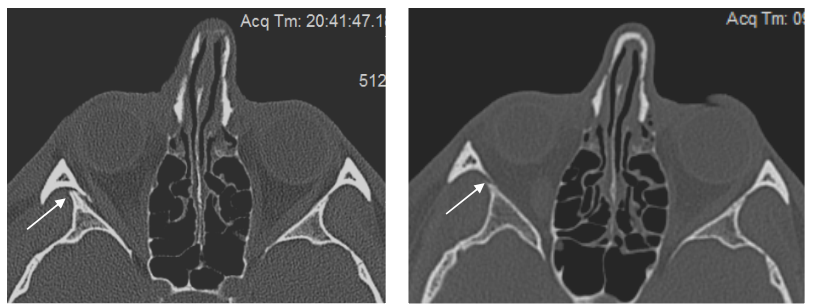

Figure 2. Computed tomography images of patient in Figure 1. Images show the zygomaticosphenoid suture (White arrow) before (a) and 9 months after (b) ZMC repair using steinmann pin.

Fractures of the zygoma can be classified based on their severity. The classification described by Zingg divided these fractures into incomplete zygomatic fractures (Type A), complete monofragmentzygomatic fracture (Type B), and multifragmentzygomatic fracture (Type C) [22]. In this classification, type B fractures include nondisplaced/minimally displaced injury to all four pillars of the malar eminence [21]. These fractures are amenable to closed reduction as long as there is no extensive disruption of the orbital floor and infraorbital rim [22]. In a review of 1025 cases of zygomatic fractures, Zingg et al., found that Type B zygomatic fractures represented approximately $57 \%$ of all fractures studied, making it the most abundant fracture type. Our series presents only type B zygomatic fractures, making them ideal for closed reduction.

Analysis of the operative time indicates a significant reduction in the time required to perform the closed reduction and external fixation repair using a Steinmann Pin. In fact, use of a Steinmann Pin reduces operative time by approximately $60 \%$, reducing exposure to general anesthesia. This is particularly helpful in geriatric patients with facial trauma.

Although there are a number of approaches that exist to repair zygomatic fractures [23-27], previous studies show that two-point fixation can provide acceptable stabilization [20]. We argue that stabilization of the zygo- matic complex to the hard palate via a Steinmann pin offers good stabilization and prevention of rotational displacement. Postoperative facial CT scans demonstrate that closed reduction with fixation using Steinmann pins provides good realignment of the zygomaticosphenoid suture (Figure 2), we recommend this technique be utilized mainly for Zingg type B fractures (noncommunited, tetrapod).

It should be mentioned that when performing a closed reduction with a Steinmann pin, only a one centimeter facial incision is required, which optimizes the patient opportunity for a satisfactory aesthetic result. In fact, based on the photographic evaluation of the patients presented here, there was excellent patient outcome with minimal scarring. As it was difficult to contact patients to evaluate their satisfaction with both repair modalities due to erroneous or outdated contact information, we will continue to find ways to obtain optimal follow-up for those patients undergoing ZMC repairs. This will allow for a detailed discussion on the patients' long-term satisfaction with either procedure.

\section{Conclusion}

Closed reduction and external fixation with a Steinmann pin of ZMC fractures provides adequate reduction of these injuries. This surgical technique results in good bony alignment and aesthetics, as measured by post-operative CT scans and patient questionnaires. Our study supports this system in the repair of trimalar fractures as it requires significantly less operating time, one small incision, and excellent patient outcomes.

\section{REFERENCES}

[1] K. Bogusiak and P. Arkuszewski, "Characteristics and Epidemiology of Zygomaticomaxillary Complex Fractures,” Journal of Craniofacial Surgery, Vol. 21, No. 4, 2010, pp. 1018-1023. doi:10.1097/SCS.0b013e3181e62e47

[2] E. H. Hagan and D. F. Huelke, "An Analysis of 319 Case Reports of Mandibular Fractures,” Journal of Oral Surgery, Anesthesia, and Hospital Dental Service, Vol. 19, 1961, pp. 93-104.

[3] W. L. Adeyemo, A. L. Ladeinde, M. O. Ogunlewe, et al. "Trends and Characteristics of Oral and Maxillofacial Injuries in Nigeria: A Review of the Literature," Head \& Face Medicine, Vol. 1, 2005, pp. 1-7. doi:10.1186/1746-160X-1-7

[4] M. R. Telfer, G. M. Jones and J. P. Shepherd, "Trends in the Aetiology of Maxillofacial Fractures in the United Kingdom (1977-1987)," British Journal of Oral and Maxillofacial Surgery, Vol. 29, No. 4, 1991, pp. 250-255. doi:10.1016/0266-4356(91)90192-8

[5] A. Alvi, T. Doherty and G. Lewen, "Facial Fractures and Concomitant Injuries in Trauma Patients," Laryngoscope, 
Vol. 113, No. 1, 2003, pp. 102-106. doi:10.1097/00005537-200301000-00019

[6] B. G. Evans and G. R. Evans, "MOC-PSSM CME Article: Zygomatic Fractures," Plastic and Reconstructive Surgery, Vol. 121, Suppl. 1, 2008, pp. 1-11. doi:10.1097/01.prs.0000294655.16607.ea

[7] K. Hwang and D. H. Kim, "Analysis of Zygomatic Fractures,” Journal of Craniofacial Surgery, Vol. 22, No. 4, 2011, pp. 1416-1421. doi:10.1097/SCS.0b013e31821cc28d

[8] Y. Kaufman, D. Stal, P. Cole, et al., "Orbitozygomatic Fracture Management,” Plastic and Reconstructive Surgery, Vol. 121, 2008, pp. 1370-1374. doi:10.1097/SCS.0b013e31821cc28d

[9] P. Kelley, R. Hopper and J. Gruss, "Evaluation and Treatment of Zygomatic Fractures,” Plastic and Reconstructive Surgery, Vol. 120, No. 7, 2007, pp. 5S-15S. doi:10.1097/01.prs.0000260720.73370.d7

[10] W. D. Appling, J. R. Patrinely and T. A. Salzer, “Transconjunctival Approach Vssubciliary Skin-Muscle Flap Approach for Orbital Fracture Repair," Archives of Otolaryngology—Head and Neck Surgery, Vol. 119, No. 9, 1993, pp. 1000-1007. doi:10.1001/archotol.1993.01880210090012

[11] J. B. Mullins, J. B. Holds, G. Branham, et al., "Complications of the Transconjunctival Approach: A Review of 400 Cases," Archives of Otolaryngology-Head and Neck Surgery, Vol. 123, No. 4, 1997, pp. 385-388.

[12] A. Baumann and R. Ewers, "Use of the Preseptal Transconjunctival Approach in Orbit Reconstruction Surgery," Journal of Oral and Maxillofacial Surgery, Vol. 59, No. 3, 2001, pp. 287-291. doi:10.1053/joms.2001.20997

[13] P. N. Manson, E. Ruas, N. Iliff, et al., "Single Eyelid Incision for Exposure of the Zygomatic Bone and Orbital Reconstruction,” Plastic and Reconstructive Surgery, Vol. 79, No. 1, 1987, pp. 120-126. doi:10.1097/00006534-198701000-00023

[14] K. Hwang, "One-Point Fixation of Tripod Fractures of Zygoma through a Lateral Browincision," Journal of Craniofacial Surgery, Vol. 21, No. 4, 2010, pp. 10421044. doi:10.1097/SCS.0b013e3181e48607

[15] B. Y. Park, S. Y. Song, I. S. Yun, D. W. Lee, D. K. Rah and W. J. Lee, "First Percutaneous Reduction and Next External Suspension with Steinmann Pin and Kirschner Wire of Isolated Zygomatic Fractures,” Journal of Craniofacial Surgery, Vol. 21, No. 4, 2010, pp. 1060-1065. doi:10.1097/SCS.0b013e3181e62cb2

[16] S. T. Kim, D. H. Go, J. H. Jung, H. E. Cha, J. H. Woo and I. G. Kang, "Comparison of 1-Point Fixation with 2-Point Fixation in Treating Tripod Fractures of the Zygoma," Journal of Oral and Maxillofacial Surgery, Vol. 69, No. 11, 2011, pp. 2848-2852. doi:10.1016/j.joms.2011.02.073
[17] B. R. Chrcanovic, Y. S. Cavalcanti and P. Reher, “Temporal Miniplates in the Frontozygomatic Area-An Anatomical Study,” Journal of Oral and Maxillofacial Surgery, Vol. 13, 2009, pp. 201-206.

[18] M. Kurita, M. Okazaki, M. Osaki, Y. Tanaka, N. Tsuji, A. Takushima and K. Harii, "Patient Satisfaction after Open Reduction and Internal Fixation of Zygomatic Bone Fractures,” Journal of Craniofacial Surgery, Vol. 21, No. 1, 2010, pp. 45-49. doi:10.1097/SCS.0b013e3181c36304

[19] Y. O. Kim, “Transcutaneous Reduction and External Fixation for the Treatment of Noncomminutedzygoma Fractures," Journal of Oral and Maxillofacial Surgery, Vol. 56, No. 12, 1998, pp. 1382-1387. doi:10.1016/S0278-2391(98)90398-6

[20] G. C. Rinehart, J. L. Marsh, K. M. Hemmer, et al., "Internal Fixation of Malar Fractures: An Experimental Biophysical Study," Plastic and Reconstructive Surgery, Vol. 84, 1989, pp. 21-25. doi:10.1097/00006534-198907000-00003

[21] E. Abemayor, J. Zemplenyi, C. Mannai, D. J. Webb and R. F. Canalis, "The Fixation of Malar Fractures with the Transnasal Kirschner Wire,” Journal of Otolaryngology, Vol. 17, No. 4, 1988, pp. 179-182.

[22] M. Zingg, K. Laedrach, J. Chen, et al., "Classification and Treatment of Zygomatic Fractures: A Review of 1025 Cases," Journal of Oral and Maxillofacial Surgery, Vol. 50, No. 8, 1992, pp. 778-790. doi:10.1016/0278-2391(92)90266-3

[23] P. R. Langsdon, T. A. Knipe, W. S. Whatley, et al., "Transconjunctival Approach to the Zygomatico-Frontal Limb of Orbitozygomatic Complex Fractures,” Facial Plastic Surgery, Vol. 21, No. 3, 2005, pp. 171-175. doi:10.1055/s-2005-922855

[24] F. Holzle, S. Swaid, T. Schiwy, et al., "Management of Zygomatic Fractures via a Transconjunctival Approach with Lateral Canthotomy While Preserving the Lateral Ligament,” Mund.Kiefer.Gesichtschir., Vol. 8, 2004, pp. 296-301.

[25] L. P. Zhong and G. F. Chen, "Subciliary Incision and Lateral Cantholysis in Rigid Internal Fixation of Zygomatic Complex Fractures," Chinese Journal of Traumatology, Vol. 7, No. 3, 2004, pp. 170-174.

[26] D. J. Courtney, "Upper Buccal Sulcus Approach to Management of Fractures of the Zygomatic Complex: A Retrospective Study of 50 Cases," British Journal of Oral and Maxillofacial Surgery, Vol. 37, No. 6, 1999, pp. 464466. doi:10.1054/bjom.1999.0010

[27] H. Matsumura, H. Yakumaru and K. Watanabe, “Temporal Approach for Reduction of Zygomatic Fractures: Clinical Results and Advantages of the Technique,” Journal of Plastic Surgery and Hand Surgery, Vol. 28, No. 1, 1994, pp. 49-53. doi:10.3109/02844319409015995 